\title{
REFLECTIONS
}

\section{A nurse's perspective on the ART rollout}

M Tito, RN, Dip Nursing Science, AHMP

Corresponding author: M Tito (mzi@iydsa.co.za)

Mzi Tito is the technical advisor for Beyond Zero, in the Nelson Mandela Bay Metropolitan, Cacadu and Harry Gwala districts of the Eastern Cape Province, South Africa

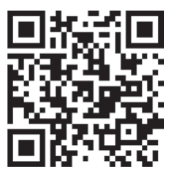

The introduction of antiretroviral therapy (ART) brought about an exciting, yet somehow scary period in terms of treatment and care in our health facilities. In 2004, when the rollout of ART started, I was fortunate to have been working in one of the first facilities to be accredited as an ART site in the Nelson Mandela Bay Metropolitan district. Only one registered nurse was trained in the ART programme; she became the 'queen' of the programme and the envy of the rest of the colleagues, as suddenly she was elevated to a specialised position. There was new terminology that we had to get used to, and the names of the new drugs were so many and complex that I thought I would never be able to pronounce them, let alone remember them. Drug names like 'stavudine' and 'lamivudine' sounded like some eastern European names!

\section{'... the names of the new drugs were so many and complex that I thought I would never be able to pronounce them, let alone remember them.'}

Early on, there were rumours making the rounds from some media and within communities that these drugs were actually very potent, toxic and dangerous to the patients - and this did little to attract some nurses to the ART programme. In fact the envy towards our trained colleagues turned to pity, because in our view, they were treading on dangerous ground (sometimes nurses can be reluctant to take professional risks for fear of medico-legal implications). Coupled with our lack of training in those early days, this ignorance instilled fear and doubt in many of us.

Yet throughout this, the number of clients receiving ART began to grow significantly. This impacted on space, as waiting areas were overflowing with ART patients. Facility managers faced several health systems challenges that had not been faced before, and planning for facilities became more difficult. This also posed a serious challenge to the facilities as nurses saw the increased patient health needs as an added burden and workload on the already overburdened staff; and this was compounded by a gross shortage of staff in general. In facilities where there was only a single nurse trained in initiation and management of ART, that person would burn out sooner or later due to increased workload. And when a lone nurse trained in nurse-initiated management of ART (NIMART) was off sick or on leave, there was often no one trained to take over from her.

\section{'... there were rumours ... that these drugs were actually very potent, toxic and dangerous to the patients - and this did little to attract some nurses to the ART programme.'}

In this context, I volunteered to become part of the ART programme. For me it was a great opportunity to become involved and I was mentored by a trained NIMART nurse while working alongside her. Working with patients receiving ART became a rewarding and fulfilling experience in my professional life. Soon I was able to breeze through the names of the drugs like an old expert! It was rewarding to see clients who were brought to the clinic unable to walk, some even in wheelbarrows, starting ART, and a few weeks later walking into the clinic unassisted and smiling. I was fortunate to work alongside a team of very capable and intelligent community health workers who had been trained in various aspects of the programme, and of course, fellow NIMART nurses who were pioneers in the programme.

Today a large number of people living with HIV are receiving $\mathrm{ART}$ and the number is growing each day. It has been incredible to witness the process unfolding right in front of my eyes, with all the excitement of seeing people's lives transformed in front of you: patients who would have died, coming back after receiving ART to live normal and healthy lives.

Ten years of ART has changed a lot of lives for the better and has impacted positively on many communities.

S Afr J HIV Med 2014;15(1):19. DOI:10.7196/SAJHIVMED.1034 